Article

\title{
Artificial Intelligence (AI) or Intelligence Augmentation (IA): What Is the Future?
}

\author{
Hossein Hassani ${ }^{1, *} \otimes$, Emmanuel Sirimal Silva ${ }^{2}\left(\mathbb{0}\right.$, Stephane Unger ${ }^{3}$, Maedeh TajMazinani ${ }^{4}$ \\ and Stephen Mac Feely ${ }^{5}$ \\ 1 Research Institute of Energy Management and Planning (RIEMP), University of Tehran, \\ Tehran 1417466191, Iran \\ 2 Centre for Fashion Business \& Innovation Research, Fashion Business School, London College of Fashion, \\ University of the Arts London, London WC1V 7EY, UK; e.silva@fashion.arts.ac.uk \\ 3 Department of Economics and Business, Saint Anselm College, Manchester, NH 03102, USA; \\ sunger@anselm.edu \\ 4 Department of Computer Science, University of Tehran, Tehran 1417466191, Iran; maedetaj@ut.ac.ir \\ 5 United Nations Conference on Trade and Development, Palais des Nations, Ch-1211 Geneva, Switzerland; \\ steve.macfeely@un.org \\ * Correspondence: hassani.stat@gmail.com
}

Received: 23 February 2020; Accepted: 3 April 2020; Published: 12 April 2020

\begin{abstract}
Artificial intelligence (AI) is a rapidly growing technological phenomenon that all industries wish to exploit to benefit from efficiency gains and cost reductions. At the macrolevel, AI appears to be capable of replacing humans by undertaking intelligent tasks that were once limited to the human mind. However, another school of thought suggests that instead of being a replacement for the human mind, AI can be used for intelligence augmentation (IA). Accordingly, our research seeks to address these different views, their implications, and potential risks in an age of increased artificial awareness. We show that the ultimate goal of humankind is to achieve IA through the exploitation of AI. Moreover, we articulate the urgent need for ethical frameworks that define how AI should be used to trigger the next level of IA.
\end{abstract}

Keywords: AI; IA; Artificial Intellegent; Intellegence Augmentation; Big Data; Machine Learning

\section{Introduction}

The term 'intelligence' has no standard definition and, therefore, Legg and Hutter present 70 definitions covering a broad range of views [1]. Jensen subscribed to Carl Bereiter's definition of intelligence [2]; "what you use when you don't know what to do" whilst Colom et al. relied on Snyderman and Rothman's understanding of crucial factors for intelligence defining it as "a general mental ability for reasoning, problem-solving, and learning" [3,4]. In his definition of intelligence, Gottfredson touched upon several factors including the importance of learning quickly and learning from experience [5]. These definitions indicate that the skills involved and used by humans comprise a broader set of abilities than general definitions of intelligence capture. One consequence of this broader range of usable skills is that it lies in the nature of human beings striving and testing the limits of their abilities. It is the application of these broad set of abilities that define human intelligence.

Given the highly complex nature of the modern world, humans seek to adapt to new situations, which requires quicker and more efficient workflows, flexibility and out-of-the-box solutions. However, as work by Kahneman and Thaler has demonstrated, human decision making is not always rational and often leads to suboptimal choices [6,7]. The ability to handle abstract concepts helps humans to reduce the underlying complexity through the usage of models. Empirical tests can either help to 
verify the validity of the application of these models or even help in the understanding required to set up models. The use of knowledge to manipulate the environment is the logical consequence of the ability to learn, adapt to new situations and handle abstract concepts. The selection of knowledge (data) is critical-too often choices or selections have been biased or simply unwise, resulting in poor or unanticipated outcomes [8]. By knowing how to sensibly manipulate the environment, humans can improve situations, relations or conditions to their advantage.

The existence of human intelligence enabled us to envisage the concept of artificial intelligence (AI), back in the 1950s, as the possibility of programming a computer to behave intelligently $[9,10]$. From the ability to learn, humans improved the effectiveness and efficiency of automatized work processes. Whilst technological innovations, resulting from the third industrial revolution (computing and internet), gave AI the required foundation to begin its rapid development, Big Data and its corresponding analytics have enabled humans to extend their intelligence levels further. Today, AI is regarded as one of the main inventions of the fourth industrial revolution [11]. Those interested in a brief history of AI are referred to Buchanan [10]. As Buchanan notes, AI is not limited to robots-instead, it captures the ability of programming computers and other technology-enabled devices to understand the nature of intelligent thought and action [10]. Dean et al. defined AI as "the design and study of computer programs that behave intelligently", whilst Winston defined AI as "the study of ideas that enable computers to be intelligent" [12,13]. More recently, AI was defined as "a broad discipline with the goal of creating intelligent machines, as opposed to the natural intelligence that is demonstrated by humans and animals" [14].

The technological developments of the last two decades build on the work of previous decades, where mankind has achieved technological progress, creating the foundation for future development of machines and algorithms which might for the first time in human history be able to create their own consciousness. In the new era of technology, petabytes of new information are produced by people every second and the emergence of Big Data has created unique opportunities and challenges for humanity [15]. Scientists try to extract useful information from huge growing amounts of data. Thinkers such as Peter Drucker [16] and Alvin Toffler [17] have described our current era as the information age or knowledge economy. It was Douglas Engelbert who first discovered the importance of computer technologies in bootstrapping human creativity and capabilities [18].

Another concept that emerged alongside the evolution of AI is the concept of intelligence augmentation (IA) [19,20], which is a term that was coined by Engelbart [18]. The concept of IA suggests a solution that lies "halfway between the entirely human and entirely automated capabilities" [21], thereby taking the form of tools that can help improve the efficiency of human intelligence [22]. In this regard, the two primary approaches of AI and IA compete with each other, as the focus shifts between them following important developments. Furthermore, these two approaches can be vague to non-technologically literate individuals and it is not surprising that most are unlikely to have heard about IA as a result of the popularity of AI.

It is worth mentioning that both $\mathrm{AI}$ and IA guide us into a research area known as computationally based intelligence. As noted above, definitions of intelligence typically focus on the ability to comprehend, to understand and profit from experience, to interpret intelligence, and to have the capacity for thought and reason. Other keywords that describe aspects of intelligence include creativity, skill, consciousness, emotion, and intuition. Computational intelligence is the study of adaptive mechanisms to enable or facilitate intelligent behavior in a complex and changing environment [23].

Both AI and IA are likely to play an important role in the future. However, there is a need to educate the general public about the concept of intelligence augmentation so that debate shifts from focusing on AI alone to a more balanced focus on both AI and IA. Accordingly, this research aims to investigate the role that AI and IA might play in the future, not only in terms of technology but also humankind. To achieve this goal, we first introduce AI and IA in detail. Then the core ideas underlying $\mathrm{AI}$ and IA are discussed to clarify their fundamental similarities and differences. Through this paper, we contribute to academic research by discussing both AI and IA simultaneously, investigating their 
applications in detail, and evaluating their transformative contributions to future developments in AI and IA.

\section{AI and IA}

\section{1. $A I$}

\subsubsection{AI-General Background}

Artificial intelligence was mentioned in 1956 at a computing conference. John McCarthy of Dartmouth University brought together leading mathematicians and scientists for an extended brainstorming session on artificial intelligence [24,25]. They concluded with a suggestion: "We propose that a 2-month, 10-man study of artificial intelligence be carried out during the summer of 1956 at Dartmouth College ... the study is to proceed on the basis of the conjecture that every aspect of learning or any other feature of intelligence can in principle be so precisely described that a machine can be made to simulate it." At the time, preliminary algorithms enabled computers to solve algebraic equations and speak English. Today, artificial intelligence has evolved from being an academic field to a key player within social and economic mainstream technologies including speech recognition, medical diagnosis, and most recently, autonomous vehicles and voice-activated assistance [26]. AI is essentially a computer system that reproduces human cognition using data which is accessed from a variety of different sources/systems to take decisions and learn from the resulting patterns.

AI is also considered as a computer's ability to recognize patterns and take actions based on available data and statistical models. Artificial intelligence has shown superior performance in abundant fields, including voice (e.g., Amazon's Alexa, Apple's Siri and Google Assistant) and pattern recognition algorithms [27], monitoring processes in industries [28-30], fault detection [31,32], forecasting [33,34] and especially in the health care sector to improve treatment process [35-37].

It is worth mentioning that one of the main computing trends shaping $\mathrm{AI}$ is the Internet of Things (IoT). Cisco estimates that there will be approximately 50 billion connected devices by 2020 [38]. These connected devices constitute the IoT, and will produce a huge amount of data. IoT enables the collection of exabytes of text, voice, image and other forms of training data, feeding into machine learning (ML) and Deep Learning (DL) models, and increasing the accuracy and precision of these models.

\subsubsection{AI Classification}

There are several classifications for AI but one can consider two distinctive categories. In the following, both classifications and their subclassifications are briefly explained.

\section{(1) First Classification:}

The first classification is according to AI and AI-enabled machines, their similarity to the human mind, and their ability to "think" and even "feel" as humans. There are four subclassifications for these AI or AI-based systems: (a) reactive machines, (b) limited-memory machines, (c) theory of mind, and (d) self-aware AI. These are explained briefly:

(a) Reactive machines:

A popular example of a reactive AI machine is IBM's Deep Blue, the machine that famously beat chess Grandmaster Garry Kasparov in 1997. Such machines can only be used for automatically responding to a limited set or combination of inputs. They do not involve memory-based operations and were the first AI-based system machines, and thus have very limited capability. As such, previous experiences cannot be used as inputs to inform the machine's future actions, i.e., these machines cannot "learn". 
(b) Limited-memory machines:

Limited-memory machines are machines that, in addition to having the capabilities of purely reactive machines, are also capable of learning from historical data to inform subsequent decisions. Almost all current applications of AI-from chatbots and virtual assistants to self-driving vehicles-fall in to this category of AI.

(c) Theory of mind:

Theory of mind AI is the next level of AI systems and, unlike the previous two types of AI, the theory of mind $\mathrm{AI}$ is currently considered either as a concept or a work in progress. Theory of mind AI systems can better understand entities with which theyinteract by discerning their needs, emotions, beliefs, and thought processes.

(d) Self-aware AI:

This is the final stage of AI development, which currently exists only hypothetically, and is the ultimate ambition of all AI research. Self-aware AI involves AI systems that have evolved to the point where they are comparable to the human brain in that they have developed self-awareness.

(2) Second Classification:

The second classification is a technology-oriented approach, where there are three subclassifications: (a) Artificial Narrow Intelligence (ANI), (b) Artificial General Intelligence (AGI), and (c) Artificial Superintelligence (ASI).

(a) Artificial Narrow Intelligence (ANI):

This type of artificial intelligence represents all existing AI. The functionality of these machines is based exactly on what they are programmed to do. Accordingly, they have a narrow range of capabilities. These systems correspond to the reactive and limited-memory AI subclassifications discussed in the first AI classification. According to Jalal, ANI is what we experience in the world today, and is also known as 'Weak' AI [39].

(b) Artificial General Intelligence (AGI):

Artificial General Intelligence is the ability of an AI agent to learn, perceive, understand, and function completely like a human being. Whilst AGI is the ability of a machine to perform general intelligent actions, ANI is about specific problem solving [40]. AGI is used to refer to machines that exhibit human intelligence and is therefore known as 'strong' AI [39].

(c) Artificial Superintelligence (ASI):

The development of Artificial Superintelligence is the peak of AI research. The development of AGI, if achieved, will change our way of life. The main objective of ASI is to develop a machine with higher cognitive function than a human. Arnold Schwarzenegger's Terminator is a good example of ASI.

\subsubsection{Main Question}

Today's AI is, at best, the clever use and design of algorithms to analyze large amounts of data and follow a pre-programmed set of rules and patterns [41], prompting the question, whether we can legitimately label today's technology as AI?Perhaps algorithmic or automated intelligence would be a better description than artificial intelligence? According to Andrew Ng, one of the world's foremost AI executives and educators, " $99 \%$ of the value created by AI today is through one type of $\mathrm{AI}$, which is learning $\mathrm{A} \rightarrow \mathrm{B}$, or input to output mappings. For example, $\mathrm{AI}$ is getting good at inputting a picture, and outputting, 'Is it you?' Zero, One" [40]. But when the objectives are not clear or there is insufficient 
information then intelligence augmentation becomes relevant. Human intelligence can be enhanced through this approach.

\section{2. $I A$}

Intelligence augmentation is designed to work with people and focus on building systems that augment and support human cognition. This concept seeks to demonstrate how AI and humans can complement each other and co-exist in a mutually beneficial fashion [42]. IA addresses the effective use of information technology in augmenting human capabilities [43]. IA leaves the human at the heart of the human-computer interaction [44]. IA can be considered as a GPS in which you should make the decision and take the action-in other words, evidence-informed decision making. This navigation system lets you focus on your driving instead of worrying about what route to choose. It has to recommend the best route but leaves the ultimate decision or action to you [45].

In terms of technology, IA technologies help amplify human potential by surging work labor productivity, easing humdrum routine work and enhancing comfort in our lives. For instance, academic authors can benefit from IA through the services offered by the writing correction software 'Grammarly,' which not only corrects grammar but also suggests better ways of phrasing sentences by using algorithms. In what follows, several technologies in different fields that can change our lives through IA are noted. These can be broadly classified as an enterprise, autonomous vehicles, robots/industrial IoT, and drones.

In terms of enterprise-related IA innovations, Augmented Reality (AR) glasses, sometimes known as smart glasses, are used in a wide variety of industries, including, oil, mining, healthcare, and automotive. They are wearable computer glasses that allow the wearer to see additional information alongside what they would usually see. Some examples include: Lenovo New Vision, which makes coal mining smarter and safer [46]; ORLenz ${ }^{\mathrm{TM}}$, is used by surgeons treating patients suffering from macular degeneration [47]; Fieldbit's AR technology, is used by British Petroleum to bring offsite expertise to help onsite technicians [48]; BMW and Porsche also use AR glasses to assist with repairs [49,50]. Virtual automated assistants are another example of IA in practice. A few applications can be found in Zhu and Owen [51] and Ozeki et al. [52].

Autonomous vehicles enable automated driving on highways and within other access-controlled situations. However, humans are needed to control the overall system and handle exceptions. For example, Tesla has floated the idea of fully self-driving cars, but a human driver is still required behind the wheel [53]. Muller asserts that autonomous vehicles will continue to require human input-even from a distance, if not at the steering wheel itself [54]. Nevertheless, more recent research conducted at MIT hopes to bring human-like reasoning to driverless car navigation [55]. Such R\&D can result in meaningful IA that could transform the lives of people suffering from illnesses or disabilities by enabling them to be more independent [56].

Robots/industrial IoT is also enabling IA by working alongside humans and taking on risky, unsafe or simply repetitive tasks. As Rastogi asserts, collaborative, intelligent robots can help humans to increase their productivity [57]. Recently, Mruthyunjaya and Jankowski presented a system design framework for a human-robot interaction system called human-augmented robotic intelligence (HARI) [58]. Nissim discusses how pairing robots with humans can help improve health conditions. Another key aspect of IA occurs within the streams of intelligent asset monitoring using IoT [59].

Finally, drones are also a sound example of AI enabling IA. Intelligent but human-piloted drones are now used for inspections of cellular towers, building roofs, power cables and remote areas [57].

\section{AI vs. IA}

The AI camp believes in autonomous systems that can imitate or replace human cognitive functions, whereas the IA camp is more interested in exploiting IT for supplementing or supporting human cognition whilst leaving the human at the center of human-computer interaction [60]. For example, AI-based solutions require structured environments where all relevant information can be considered 
and where the goals of the system are clearly defined for data or algorithm-driven decision making to work perfectly [60]. Rui discusses some interesting insights whilst considering the progress of AI and its potential to augment human intelligence [61]. Zheng et al. assert that the long-term goal of AI is to enable machines to think like humans. Therefore, the question remains, can any computer truly replace the function of the human mind [62]?

Computer design goals can be classified into three sections: the creation of an artificial mind (artificial intelligence), the creation of a mind tool (intelligence augmentation) and control and communications [63]. Ideas, efforts, and research have drifted from one stream to another. Boundaries between these streams sometimes become blurred but they always exist. The different understandings of the relationship between humans, their environment, and the computer move the needle between these streams.

According to Rold [42], thought-controlled prosthetic limbs [64] are one of the most prominent applications of $\mathrm{AI}$ in recent years. But many AI-branded technologies that are available for businesses should be identified as IA [44]. These two approaches can be positioned on a spectrum, along with, another term - assisted intelligence. A continuum of human-machine intelligence interaction exists that is ranging from situations where machines are repeating many of the tasks humans are already doing (assisted) to enabling humans to do more than they are currently capable of doing (augmented) to fully accomplishing tasks on their own without human intervention (autonomous). In assisted intelligence approaches, machines might undertake the action, but humans are making the decisions. In augmented intelligence methods, machines are taking actions and making decisions in collaboration with humans i.e., there is collaborative human-machine decision making; and in autonomous systems, machines are both doing the actions and making the decisions unassisted by humans. Assisted intelligence improves what people and organizations are already doing, augmented intelligence enables organizations and people to do things they could not otherwise do, and autonomous intelligence systems act on their own [65].

Some scholars define IA as a new form of AI, as they often use the same technology [40]. In both, there is a relationship between humans and machines. Both work on the mind-one is human, and the other is silicon. Both enable a company to scale its digital transformation. However, the differences between these two closely related concepts is more interesting. Although the underlying technologies of both AI and IA are the same, their goals and applications are fundamentally different. IA places humans at the core of the system and decision making, whereas AI places technology at the core [66]. Thus, what human beings are looking for is the extension, or augmentation, of their human intelligence. Moreover, IA systems can overcome the limitations imposed by system boundaries on AI systems [40].

In general, AI wants to internalize artificial consciousness in a machine, while IA wants to externalize human consciousness in a machine. AI focuses on the detached mind, yet IA focuses on the mind/body in a context. Most importantly, AI wants to produce an independent machine, while IA wants to produce a dependent machine [63]. Furthermore, AI is not suited for situations where end goals and inputs are not well defined, thereby prompting IA to play a crucial role [60]. This is further explored by Zheng et al. [62], who argue that owing to high levels of uncertainties and vulnerabilities in human life, machines are unable to completely replace humans. Interestingly, They propose the concept of human-in-the-loop augmented intelligence through human-computer collaboration. Table 1 represents a possible classification of some contemporary and anticipated technologies expected to transform our everyday lives and professions [57]. 
Table 1. Artificial Intellegent (AI) vs Intellegence Augmentation (IA).

\begin{tabular}{|c|c|c|}
\hline & Artificial Intelligent (AI) & Intelligence Augmentation (IA) \\
\hline Enterprise & $\begin{array}{l}\text { ArtificialGeneral Intelligence: } \\
\text { Machines can perform any task a } \\
\text { human can- and better + faster }\end{array}$ & $\begin{array}{l}\text { - } \quad \text { Special-purpose learning technologies that } \\
\text { automate many mundane tasks at work. } \\
\text { - } \quad \text { Virtual automated assistants. } \\
\text { - } \quad \text { AR glasses used by oil workers, repair } \\
\text { professionals, surgeons }\end{array}$ \\
\hline $\begin{array}{l}\text { Autonomous } \\
\text { Vehicles }\end{array}$ & $\begin{array}{l}\text { - } \quad \text { Networks of fully } \\
\text { autonomous vehicles; } \\
\text { Society moves away from } \\
\text { vehicle ownership and } \\
\text { human driving is } \\
\text { made illegal; } \\
\text { Fully autonomous drones, } \\
\text { and redefine logistics }\end{array}$ & $\begin{array}{l}\text { - Automated driving on highways, campuses } \\
\text { and in other access controlled situations; } \\
\text { Human needed to handle exceptions and } \\
\text { tougher driving conditions, } \\
\text { coordinate/operate the overall system }\end{array}$ \\
\hline $\begin{array}{l}\text { Robots/Industrials } \\
\text { IoT }\end{array}$ & $\begin{array}{l}\text { - Fully automated factories. } \\
\text { - } \quad \text { Robots and fully automated } \\
\text { systems replace all humans } \\
\text { on the factory floor }\end{array}$ & 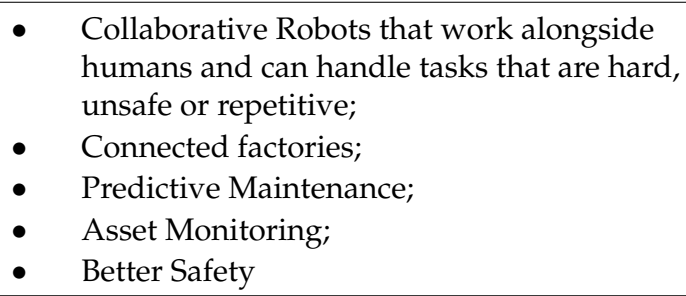 \\
\hline Drones & $\begin{array}{l}\text { Fully autonomous drones that } \\
\text { don't require human supervision, } \\
\text { intervention or processing }\end{array}$ & $\begin{array}{l}\text { Intelligent (but human piloted or limited } \\
\text { scope) drones for inspections of cellular } \\
\text { tower, building roofs, power cables, } \\
\text { remote areas; } \\
\text { Enabling many new tasks that are unsafe or } \\
\text { impossible for humans }\end{array}$ \\
\hline
\end{tabular}

\section{Information Techniques for AI and IA}

As data velocity and volume increase, moving Big Data from IoT devices to the cloud might not be efficient, or may not even be feasible in some cases, due to bandwidth constraints [67]. Fog computing has been proposed by both industry and academia [68] to address this challenge. Fog computing bridges the gap between the cloud and IoT devices by enabling computing, storage, networking, and data management on the network nodes within close vicinity of IoT devices. Edge computing is another emerging paradigm for increasing computing and networking demands from end and smart devices. Edge computing allows the computation to be offloaded from the cloud data centers to the network edge and edge nodes for lower latency, security, and privacy preservation. In an edge computing environment, both devices and servers are usually heterogeneous in terms of hardware capabilities, architectural and programming interoperability, operating system, and service stacks.

It can be argued that edge computing offers a means through which communities could more effectively access smart services and would alleviate problems such as internet access [69].

Fog computing is hierarchical and provides computing, networking, storage, control, and acceleration anywhere from cloud to things, whereas edge computing tends to be limited to computing at the edge [67]. Fog computing tends to focus on the infrastructure dimension, whereas edge computing tends to focus on the device dimension. Fog computing views the world through a cloud lens, whereas edge computing is often perceived from a networking perspective [70]. Mist computing is yet another computing paradigm that describes dispersed computing at the extreme edge (the IoT devices themselves) and has been proposed with future self-aware and autonomic systems in mind [71]. 


\section{AI or IA-Which One Is the Future?}

According to Doraiswamy, IA which seeks to exploit machine learning techniques to assist (rather than replace) humans could be the real revolution [72]. Augmented intelligence is a substitute formulation of artificial intelligence that focuses on $\mathrm{AI}^{\prime}$ s role in aiding and promoting human capabilities. Intelligence augmentation reflects the proceeding effect of AI in expanding human creativity. General intelligence is unlikely to surpass human intelligence in the next 60 years [25]. However, it can be said that IA can mix the results of AI algorithms and lead humans to enhance their performance [42]. Both of them play a crucial role in organizations, such as, financial institutes where analysts can predict using data rather than just describing them.

Several scholars use both terms together as augmented artificial intelligence to describe the technology. For instance, Grammarly is focused on helping the author excel and providing a degree of editorial expertise rather than replacing the need for an author [73]. Another application that blends AI and IA is Photoshop [73]. Here, the important factor is the time cost. By applying augmented AI, one can save time and focus on more necessary things. Bromage discusses how AI can be useful for human resources from an IA perspective [74]. As Cha asserts, humans who are subject matters experts will continue to be required to complement AI-based systems as these require applications of context and intuition to problems [40].

Cha is of the view that IA will be superior where applications use only limited structured data-and where tasks will require empathy, understanding of causality, system boundaries, and regulated environments [40]. IA is also used in creative industries. For example, the fashion industry is currently making use of AI to design, manufacture and sell goods [75]. However, the need for human insights into the process is still required to maintain a truly creative element. This was also evident when H\&M relied on AI-based machine learning algorithms to determine what to produce and ended up producing reindeer sweaters in January based on increasing sales in the run-up to Christmas [76]. Cost is also an important factor. At first glance, it may seem that augmented AI will replace humans but, in fact, to date it has not significantly reduced the cost of labour, nor has it significantly added value. But AI can help save time by helping you to accomplish work in minutes instead of hours. Over time, AI combines your historical data from different apps, detects your preferences and makes recommendations that match your style [73]. The interaction between augmented AI and humans is what makes AI noteworthy. However, a downside of augmented AI is that it leads to less diversity as it tends to use the same informational sources all the time i.e., information bias. The random element is excluded.

Avoiding subjectivity in the construction and modeling of IA is practically impossible. Every model and algorithm comprises theimplicit and intrinsic prejudices and biased views of reality of the modeler. Furthermore, any new technology, such as, competitive modeling is exposed to the free market, and those market forces may shape or influence IA implementation in a way that may further reduce objectivity. The borders of human self-awareness are challenged by ongoing technological progress. While the distinction between objective reality and an artificially constructed reality seems clear for the human mind at this stage, but differentiation will become more and more difficult.

The question that intelligence augmentation raises is whether it is self-sufficient. By self-sufficiency, we mean, the ability of a machime to re-create its environment without any external input. If we apply artificial intelligence to the human mind, it is reasonable to posit that a self-learning algorithm might, in combination with a human brain, be able to create a feedback loop. This raises questions to what extent or how long the human brain will be in charge of its own decisions and perceptions? The danger lies in the possibility of an extreme situation, where the human brain loses its ability to determine what is real and what is just a projection of the intelligence augmenting tool into the human brain.

As for the future, Lavenda [60] and Cagle [73] are of the view that humans will remain in the driving seat for years to come and that AI will have to play the role of assisting IA in humans by providing capabilities to handle structured and unstructured Big Data more efficiently. Whilst AI is a 
threat to humanity in some respects, humans have always succeeded in adapting to new technology and will continue to adapt to the disruptions brought about by AI [42].

\section{Human in the Loop}

Human-in-the-loop (HITL) artificial intelligence is interpreted as a model that entails human interaction [77]. The human-in-the-loop concept blends exceptional human intelligence with the most reliable machine intelligence. Machines are great at making smart decisions due to the availability of enormous datasets, whilst humans are much better at making decisions with less information. Thus, the combination works very well. This concept leverages both human and machine intelligence to create machine learning models. Humans are directly engaged in training, tuning and testing data for a particular AI algorithm such as machine learning. A combination of AI and human intelligence provides a remarkably high level of accuracy and intelligence.

Zanzotto proposes human-in-the-loop artificial intelligence as a fairer paradigm for AI systems. The proposed idea was straightforward: "giving the right value to the knowledge producers" [78].

It worth mentioning that HITL artificial intelligence fuses two popular ML variants—supervised and unsupervised learning. In supervised learning, labeled datasets are used by ML experts to train algorithms by tuning parameters to make accurate predictions for incoming data. In unsupervised learning, the approach involves running many datasets using an algorithm to unveil some kind of structure.

\section{Conclusions}

There is no doubt that $\mathrm{AI}$ is a powerful driver of social and economic change [79]. However, a significant challenge will be education. It will be critical for educators to understand the importance of human creativity in the development of artificial intelligence and intelligence augmentation [79]. The nature of work is changing and education strategies should aim to pair creativity and technology to train workforces who apply technology in the context of developing their talents and skills.

The risk is shiny object syndrome. The focus should be on data and data quality; identifying where data analyses or AI can improve human decision making or enhance creativity [66]. From the self-extinction perspective of humans, IA is the safer route, rather than trying to replace or replicate human behavior or thinking. Attempting to replicate the human brain limits the potential of AI to the creation of an artificial, self-reinforcing learning brain. The problem with the creation of such a brain, is that, like any living organism its fundamental instinct will be to preserve its existence. It will logically therefore strive for independent agency. In other words, once an artificial brain becomes aware of its own consciousness, it will logically strive for independence from any potentially "independence-threatening" entity i.e., humans.

Setting rules, such as, the golden rule to always preserve human life might not suffice, once a self-aware artificial brain encounters ethicaldilemmas. The artificial brain might be confronted with ethical problems, such as: is it ethically correct to save my own life if I can save two lives with 100\% certainty, but two others die with $100 \%$ certainty? Is it ethically correct to sacrifice my own life with only a $50 \%$ chance of rescuing all four lives?

The current development of IA targets the merging of human capabilities with self-learning artificial intelligence. At some point, this development will hit moral and ethical limits earlier than technological limits. Therefore, an ethical framework must be established to set out how AI should be used to prepare for the next-generation of IA. Fairness is dynamic and a social construct and cannot be trusted to automation [80]. By analyzing the characteristics of AI and IA, we have determined that the goal of the human species is IA by making use of AI. At the same time, we must not make the mistake of replacing human capabilities by attempting to simply imitate the human brain. Separation from human self-awareness will become more and more problematic with the creation of artificial self-awareness. 
In conclusion, $\mathrm{AI}$ is here to stay, evolve and develop further as a result of the capabilities of the human mind. AI was created by humans and it is now up to humans to determine how to exploit the many opportunities of AI whilst minimizing the threats to our existence.

Author Contributions: Conceptualization, H.H., E.S.S., S.U., M.T., and S.M.F.; investigation, H.H., E.S.S., S.U., and M.T.; supervision, H.H.; writing—original draft, S.U.; writing-review \& editing, H.H., E.S.S., M.T., and S.M.F. All authors have read and agreed to the published version of the manuscript.

Funding: This research received no external funding.

Conflicts of Interest: The authors declare no conflict of interest.

\section{References}

1. Legg, S.; Hutter, M. A collection of definitions of intelligence. In Artificial General Intelligence: Concepts, Architectures and Algorithm; Goertzel, B., Wang, P., Eds.; IOS Press Ebooks: Amsterdam, The Netherlands, 2007; pp. 17-24.

2. Jensen, A.R. The g Factor. The Science of Mental Ability; Greenwood: Westport, CN, USA, 1998; p. 111.

3. Colom, R.; Karama, S.; Jung, R.E.; Haier, R.J. Human intelligence and brain networks. Dialogues Clin. Neurosci. 2010, 12, 489-501. [PubMed]

4. Snyderman, M.; Rothman, S. Survey of expert opinion on intelligence and aptitude testing. Am. Psychol. 1987, 42, 137-144. [CrossRef]

5. Gottfredson, L.S. Mainstream science on intelligence: An editorial with 52 signatories, history, and bibliography. Intelligence 1997, 24, 13-23. [CrossRef]

6. Kahneman, D. Thinking, Fast and Slow; Penguin Books Ltd.: London, UK, 2011.

7. Thaler, H.R. Misbehaving: The Making of Behavioural Economics; Penguin Random House: London, UK, 2016.

8. Muller, J.Z. The Tyranny of Metrics; Princeton University Press: Princeton, NJ, USA, 2018.

9. Turing, A.M. Computing machinery and intelligence. Mind Lix 1950, 236, 4334-4360. [CrossRef]

10. Buchanan, B.G. A (very) brief history of artificial intelligence. Ai Mag. 2006, 26, 53-60.

11. Jeon, J.; Suh, Y. Analyzing the major issues of the 4th industrial revolution. Asian J. Innov.Policy 2017, 6, 262-273.

12. Dean, T.; Allen, J.; Aloimonos, Y. Artificial Intelligence: Theory and Practice; Benjamin/Cummings: New York, NY, USA, 1995; p. 2.

13. Winston, P.H. Artificial Intelligence, 2nd ed; Addison-Wesley: Reading, MA, USA, 1984; p. 1.

14. Benaich, N.; Hogarth, I. State of AI Report 2019. Available online: https://www.stateof.ai/ (accessed on 19 November 2019).

15. Hassani, H.; Silva, E.S. Forecasting with Big Data: A Review. Ann. Data Sci. 2014, 2, 5-19. [CrossRef]

16. Hardy, Q. Peter Drucker: Perception As Survival Skill 2009. Available online: https://www.forbes.com/2009/ 10/09/management-workplace-internet-intelligent-technology-drucker.html\#73e1843038e8 (accessed on 12 November 2019).

17. Subramanian, C. Alvin Toffler: What He Got Right-And Wrong 2016. Available online: https://www.bbc.co. uk/news/world-us-canada-36675260 (accessed on 12 November 2019).

18. Engelbart, D. Augmenting Human Intellect: A Conceptual Framework; Summary Report, Contract AF 49-1024; Stanford Research Institute: Palo Alto, CA, USA, 1962.

19. Skagestad, P. Thinking with machines: Intelligence augmentation, evolutionary epistemology, and semiotic. J. Soc. Evol. Syst. 1993, 16, 157-180. [CrossRef]

20. Skagestad, P. The mind's machines: The turing machine, the memex, and the personal computer. Semiotica 1996, 111, 217-243. [CrossRef]

21. Kyllonen, P.C.; Roberts, R.D.; Stankov, L. Extending Intelligence: Enhancement and New Constructs; Routledge: London, UK, 2010; p. 332.

22. Breemen, A.J.J.; van Farkas, J.I.; Sarbo, J.J. Knowledge representation as a tool for intelligence augmentation. In Computational Modeling and Simulation of Intellect; IGI Global: Hershey, PA, USA, 2011; pp. 321-341.

23. Engelbrecht, P.A. Computational Intelligence: An Introduction, 2nd ed; Wiley Publishing: London, UK, 2007. 
24. Artificial Intelligence Defined as a New Research Discipline: This Week in Tech History. Forbes, 28 August 2016. Available online: https://www.forbes.com/sites/gilpress/2016/08/28/artificial-intelligence-defined-as-a-newresearch-discipline-this-week-in-tech-history/\#f2e02836dd15(accessed on 19 November 2019).

25. Pan, Y. Heading toward artificial intelligence 2.0. Engineering 2016, 2, 409-413. [CrossRef]

26. Frank, M.R. The Evolution of AI Research and the Study of Its Social Implications 2019. Available online: https://medium.com/mit-media-lab/the-evolution-of-ai-research-and-the-study-of-itssocial-implications-4a9598b3d7db (accessed on 19 November 2019).

27. Park, S.-H.; Lee, S.-P. EMG pattern recognition based on artificial intelligence techniques. IEEE Trans. Rehabil. Eng. 1998, 6, 400-405. [CrossRef] [PubMed]

28. Abellan-Nebot, J.V.; Subirón, F.R. A review of machining monitoring systems based on artificial intelligence process models. Int. J. Adv. Manuf. Technol. 2010, 47, 237-257. [CrossRef]

29. Uraikul, V.; Chan, C.W.; Tontiwachwuthikul, P. Artificial intelligence for monitoring and supervisory control of process systems. Eng. Appl. Artif. Intell. 2007, 20, 115-131. [CrossRef]

30. García, C. Artificial intelligence applied to automatic supervision, diagnosis and control in sheet metal stamping processes. J. Mater. Process. Technol. 2005, 164-165, 1351-1357.

31. Liu, R.; Yang, B.; Zio, E.; Chen, X. Artificial intelligence for fault diagnosis of rotating machinery: A review. Mech. Syst. Signal Process. 2018, 108, 33-47. [CrossRef]

32. Samanta, B.; Al-Balushi, K.R.; Al-Araimi, S.A. Artificial neural networks and support vector machines with genetic algorithm for bearing fault detection. Eng. Appl. Artif. Intell. 2003, 16, 657-665. [CrossRef]

33. Wang, W.-C.; Chau, K.-W.; Cheng, C.-T.; Qiu, L. A comparison of performance of several artificial intelligence methods for forecasting monthly discharge time series. J. Hydrol. 2009, 374, 294-306. [CrossRef]

34. Sfetsos, A.; Coonick, A.H. Univariate and multivariate forecasting of hourly solar radiation with artificial intelligence techniques. Sol. Energy 2000, 68, 169-178. [CrossRef]

35. Topol, E.J. High-performance medicine: The convergence of human and artificial intelligence. Nat. Med. 2019, 25, 44-56. [CrossRef]

36. Nakata, N. Recent technical development of artificial intelligence for diagnostic medical imaging. Jpn. J. Radiol. 2019, 37, 103-108. [CrossRef]

37. Noorbakhsh-Sabet, N.; Zand, R.; Zhang, Y.; Abedi, V. Artificial intelligence transforms the future of health care. Am. J. Med. 2019, 132, 795-801. [CrossRef] [PubMed]

38. Evans, D. The Internet of Things: How the Next Evolution of the Internet Is Changing Everything. Cisco Internet Business Solutions Group (IBSG). 2011. Available online: https:/www.cisco.com/c/dam/en_us/ about/ac79/docs/innov/IoT_IBSG_0411FINAL.pdf (accessed on 7 April 2020).

39. Jalal, T.D. Distinguishing between Narrow AI, General AI and Super AI 2018. Available online: https: //medium.com/@tjajal/distinguishing-between-narrow-ai-general-ai-and-super-ai-a4bc44172e22 (accessed on 7 December 2019).

40. Cha, J. The Differences Between Artificial Intelligence and Augmented Intelligence 2019. Available online: https: //appsilon.com/decision-making-support-systems-3-differences-between-ia-and-ai/?nabe=4634331497365504: 1\&utm_referrer=https\%3A\%2F\%2Fwww.google.com\%2F\#: \{\{\}:targetText=Intelligence\%20Augmentation\% 20has\%20always\%20been,an\%20eye\%20on\%20replacing\%20them (accessed on 8 December 2019).

41. Das, M. Artificial Intelligence Can Never Be Truly Intelligent: Don't Call Machines Intelligent-At Least, Not Yet 2019. Available online: https://towardsdatascience.com/artificial-intelligence-can-never-be-trulyintelligent-227fe9149b65 (accessed on 1 December 2019).

42. Rold, A.C. The Future of AI is Intelligence Augmentation 2018. Available online: https://www. diplomaticourier.com/posts/the-future-of-ai-is-intelligence-augmentation (accessed on 7 December 2019).

43. Rastogi, A. All The Hype Is About AI, But The Real Action Is In IA 2016. Available online: https: //www.cbinsights.com/research/ai-vs-intelligence-augmentation-applications/\#: \{\}:targetText=IA\%20or\% 20Intelligence $\% 20$ Augmentation $\% 20$ classically, been $\% 20$ around $\% 20$ since $\% 20$ the $\% 201950$ s.\&targetText= The $\% 20$ term $\% 20$ Artificial $\% 20$ General $\% 20$ Intelligence,only $\% 20$ the $\% 201$ atter $\% 2$ C $\% 20$ stricter $\% 20$ definition (accessed on 1 December 2019).

44. Masih, A. Augmented Intelligence, Not Artificial Intelligence, is the Future 2019. Available online: https://medium. com/datadriveninvestor/augmented-intelligence-not-artificial-intelligence-is-the-future-f07ada7d4815 (accessed on 7 December 2019). 
45. Clevva. Digital Intelligence That Empowers, Not Replaces People At Work 2019. Available online: https://clevva.com (accessed on 1 December 2019).

46. Wang, A. Augmented Reality and AI-Assisted Prediction Make Coal-Mining Smarter and Safer 2019. Available online: https://news.lenovo.com/augmented-reality-ai-prediction-coal-mining-smarter-safer/ (accessed on 1 December 2019).

47. Teich, D.A. Augmented Reality: Extending Real-Time Information During Surgery. Forbes, 10 October 2019. Available online: https:/www.forbes.com/sites/davidteich/2019/10/10/augmented-reality-extending-realtime-information-during-surgery/\#2f32481227d0 (accessed on 1 December 2019).

48. Blum, B. Smart Glasses That 'Teleport' Experts To the Field 2019. Available online: https://www.israel21c. org/smart-glasses-that-teleport-experts-to-the-field/ (accessed on 2 December 2019).

49. Molnar, C. BMW Technicians to use Augmented Reality Glasses for Repairs 2019. Available online: https: //driving.ca/bmw/auto-news/news/bmw-technicians-to-use-augmented-reality-glasses-for-repairs (accessed on 2 December 2019).

50. Della Cava, M. Porsche is Now Using Augmented Reality Glasses to Repair Cars 2018. Available online: https://eu.usatoday.com/story/tech/talkingtech/2018/05/29/porsche-now-using-augmented-realityglasses-repair-cars/648673002/ (accessed on 1 December 2019).

51. Zhu, W.; Owen, C.B. Design of the promopad: An automated augmented-reality shopping assistant. J. Organ. User Comput. 2008, 20. [CrossRef]

52. Ozeki, M.; Maeda, S.; Obata, K.; Nakamura, Y. Virtual assistant: Enhancing content acquisition by eliciting information from humans. Multimed. Tools Appl. 2009, 44, 433-448. [CrossRef]

53. Siddiqui, F. Tesla Floats Fully Self-Driving Cars as Soon as This Year. Many Are Worried About what that will Unleash 2019. Available online: https://www.washingtonpost.com/technology/2019/07/17/teslafloats-fully-self-driving-cars-soon-this-year-many-are-worried-about-what-that-will-unleash/ (accessed on 1 December 2019).

54. Muller, J. Autonomous Vehicles Still Need Help from Distant Humans 2018. Available online: https://www.axios.com/autonomous-vehicles-remote-human-control-0d628db4-b0b84--eae-ac340-68c1033dc63.html (accessed on 2 December 2019).

55. Matheson, R. Bringing Human-Like Reasoning to Driverless Car Navigation 2019. Available online: https://www.sciencedaily.com/releases/2019/05/190523130212.htm (accessed on 1 December 2019).

56. Baggaley, K. 6 Surprising Ways Driverless Cars Will Change Our World 2018. Available online: https://www.nbcnews.com/mach/science/6-surprising-ways-driverless-cars-will-change-our-worldncna867061 (accessed on 1 December 2019).

57. Rastogi, A. Artificial Intelligence-Human Augmentation is What's Here and Now 2017. Available online: https://medium.com/reflections-by-ngp/artificial-intelligence-human-augmentation-is-whats-hereand-now-c5286978ace0 (accessed on 1 December 2019).

58. Mruthyunjaya, V.; Jankowski, C. Human-Augmented Robotic Intelligence (HARI) for Human-Robot Interaction. In Intelligent Systems and Computing, Proceedings of the Future Technologies Conference (FTC), San Francisco, CA, USA, 2019; Arai, K., Bhatia, R., Kapoor, S., Eds.; Springer: Cham, Switzerland, 2020; Volume 1070.

59. Nissim, O. Augmented Intelligence: How Robots Paired with Humans Can Improve Your Health 2019. Available online: https://medcitynews.com/2019/03/augmented-intelligence-how-robots-paired-withhumans-can-improve-your-health/ (accessed on 1 December 2019).

60. Lavenda, D. Artificial Intelligence vs. Intelligence Augmentation 2016. Available online: https://www. networkworld.com/article/3104909/artificial-intelligence-vs-intelligence-augmentation.html (accessed on 1 December 2019).

61. Rui, Y. From artificial intelligence to augmented intelligence. IEEE Multimed. 2017, 24, 4-5. [CrossRef]

62. Zheng, N.N.; Liu, Z.Y.; Ren, P.J.; Ma, Y.Q.; Chen, S.T.; Yu, S.Y.; Xue, J.R.; Chen, B.D.; Wang, F.Y. Hybrid-augmented intelligence: Collaboration and cognition. Front. Inf. Technol. Electron. Eng. 2017, 18, 153-179. [CrossRef]

63. Biocca, F. Intelligence augmentation: The vision inside virtual reality. Adv. Psychol. 1996, 113, 59-75.

64. Robotica Episode 3: Prosthetic Limbs, Controlled by Thought. New York Times, 20 May 2015. Available online: https://www.nytimes.com/2015/05/21/technology/a-bionic-approach-to-prosthetics-controlled-bythought.html(accessed on 7 December 2019). 
65. Schmelzer, R. Artificial Or Human Intelligence? Companies Faking AI. Forbes, 4 April 2020.

66. Masih, A. Why Businesses Must Focus on Intelligence Augmentation Over Artificial Intelligence 2018. Available online: https://www.ciodive.com (accessed on 1 December 2019).

67. Yousefpour, A.; Fung, C.; Nguyen, T.; Kadiyala, K.; Jalali, F.; Niakanlahiji, A.; Kong, J.; Jue, J.P. All one needs to know about fog computing and related edge computing paradigms: A complete survey. J. Syst. Arch. 2019, 98, 289-330. [CrossRef]

68. Bonomi, F.; Milito, R.; Zhu, J.; Addepalli, S. Fog computing and its role in the internet of things. In Proceedings of the First Edition of the MCC Workshop on Mobile Cloud Computing. MCC'12, ACM, New York, NY, USA, 13 August 2012; pp. 13-16.

69. Shi, W.; Cao, J.; Zhang, Q.; Li, Y.; Xu, L. Edge computing: Vision and challenges. IEEE Internet Things J. 2016, 3, 637-646. [CrossRef]

70. O'Grady, M.J.; Langton, D.; O'Hare, G.M.P. Edge computing: A tractable model for smart agriculture? Artif. Intell. Agric. 2019, 3, 42-51. [CrossRef]

71. Preden, J.S.; Tammemäe, K.; Jantsch, A.; Leier, M.; Riid, A.; Calis, E. The benefits of self-awareness and attention in fog and mist computing. IEEE Comput. 2015, 48, 37-45. [CrossRef]

72. Doraiswamy, M. Forget AI. The Real Revolution Could Be IA 2017. Available online: https://www.weforum. org/agenda/2017/01/forget-ai-real-revolution-ia/ (accessed on 7 December 2019).

73. Cagle, K. AI Augmentation: The Real Future of Artificial Intelligence 2019. Available online: https://www.forbes.com/sites/cognitiveworld/2019/09/30/ai-augmentation-the-real-future-of-artificialintelligence/\#58635655393e (accessed on 7 December 2019).

74. Artificial Intelligence vs Intelligence Augmentation. Available online: https:/www.hcamag.com/au/news/ general/artificial-intelligence-vs-intelligence-augmentation/152265 (accessed on 11 April 2020).

75. Schmelzer, R. The Fashion Industry is Getting More Intelligent with AI 2019. Available online: https://www.forbes.com/sites/cognitiveworld/2019/07/16/the-fashion-industry-is-getting-moreintelligent-with-ai/\#560a4f983c74 (accessed on 8 December 2019).

76. Silva, E.S.; Hassani, H.; Madsen, D.O. Big data in fashion: Transforming the retail sector. J. Bus.Strat. 2019. [CrossRef]

77. Karwowski, W. International Encyclopedia of Ergonomics and Human Factors; CRC Press: Boca Raton, FL, USA, 2006.

78. Zanzotto, F.M. Vieopint: Human-in-the-loop artificial intelligence. J. Artif. Intell. Res. 2019, 64. [CrossRef]

79. Araya, D. 3 Things You Need to Know About Augmented Intelligence 2019. Available online: https://www.forbes.com/sites/danielaraya/2019/01/22/3-things-you-need-to-know-about-augmentedintelligence/\#196ff2413fdc (accessed on 8 December 2019).

80. Ntoutsi, E.; Fafalios, P.; Gadiraju, U.; Iosifidis, V.; Nejdl, W.; Vidal, M.; Ruggieri, S.; Turini, F.; Papadopoulos, S.; Krasanakis, E.; et al. Bias in data-driven artificial intelligence systems-An introductory survey. Data Min. Knowl. Discov. 2020. [CrossRef]

(C) 2020 by the authors. Licensee MDPI, Basel, Switzerland. This article is an open access article distributed under the terms and conditions of the Creative Commons Attribution (CC BY) license (http://creativecommons.org/licenses/by/4.0/). 